\title{
A COMMENTARY BY REMIGIUS AUTISSIODORENSIS ON THE DE CONSOLATIONE PHILOSOPHIAE OF BOETHIUS.
}

As long ago as I 88I, and again in I894, the late Dr Georg Schepss, whom students of Boethius must always remember with respect and regret, pointed to the unharvested field of Latin scholia on the De consolatione philosophiae and called for labourers. ${ }^{1}$ 'These glosses, which are a familiar feature of Boethius manuscripts, not only help, as Schepss shewed, to explain many of the eccentricities of mediaeval translators - and imitators, ${ }^{8}$ but also raise some interesting questions of origin and authorship. To unravel successfully the different commentaries, which by the tenth and eleventh centuries had become a regular tangle, would bring a reward proportionate to the difficulty of the task. So far as I am aware the problem has been seriously attacked by no one, although Professor E. K. Rand, of Harvard, touched it lightly, but with a sure hand, in the course of his brilliant study of the commentaries on the Opuscula sacra. In the volume of Traube's Quellen und Unters. sur lat. Philologie des Mittelalters entitled Jokannes Scottus, noteworthy as bringing to light an unknown work of the great Irishman, Professor Rand mentions a Trèves manuscript (I093) of the eleventh century, on fol. $115^{\nabla}$ of which is the rubric: INCIPIT ExPósItio IN LIBRO BOETII De consolatione Philosophiae Remigi Autisiodoriensis magistri. Upon this there follow two of the usual Vitae Boetii, and then the text of the De consolatione philosophiae, accompanied by a very full commentary, marginal and interlinear. Professor Rand at once noticed the mixed character of the commentary, and opined, no doubt correctly, that the better glosses were borrowings and not original contributions. But his chief business being with the Opuscula sacra, he did not

1 Handschrifliche Studien eu Boethius: Programm Wurzburg 1881; Wochenschrift fü kl. Philologie April II, 1894.

2 These curiosities hardly fall within the scope of the J.T.S., but to whet the reader's interest 1 may mention that Remigius is apparently accountable for the mediaeval fancy that Alcibiades was a woman (cf. Villon, Ballads des dames du temps jadis, 'Archipiades'), and for a knowledge of the name of Croesus's daughter 'Fania' (cf. Chaucer Morks Tale 3917, Romar de la Rase 7232-7258), and that 'Mythographus Vaticanus II' is quite possibly none other than the monk of Auxerre. (See Manitius Geschichte der lat. Litt. des Mittelalters p. 509).

' Cf. below, p. 4 n. 2. 
embark on a detailed examination of a manuscript from which these were absent. By the kindness of the librarian at Trèves the codex was placed at my disposal in Cambridge for three months last year, and I was able to examine it at leisure. While I was looking for some clue which should enable me to differentiate the work of Remi from his predecessors', I happened on words which were plainly not

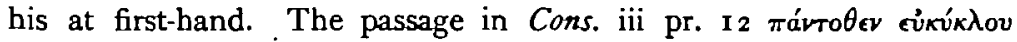

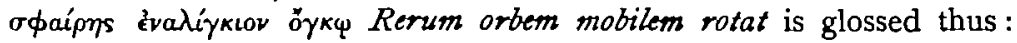
Secundum commentum Remigii explanatio graeci uersus deest quia penitus corruptus est. Now the same gloss, without the qualification secundum commentum Remigii, occurs in a continuous commentary contained in an eleventh-century manuscript of the Öttingen-Wallerstein collection at Maihingen, with which Schepps dealt minutely in his Programm, calling it $K$, and of which I possess a photograph. The suspicion raised by this discovery became a conviction when $I$ found that practically the whole of the continuous commentary could be extracted from the margin or from between the lines of the Trèves manuscript (which I call $L$ ). The obvious conclusion, borne out by other internal evidence, is that $K$ is what $L$ claims to be, viz. the Commentary of Remigius. Acting upon this assumption I transcribed $K$, controlling its very defective readings (the manuscript is evidently written by a novice) by reference to $L$ and other manuscripts, of which there is no lack in Cambridge, London, and Paris. I had hoped to print the commentary in extenso this year, and projected a visit to Trèves and Munich last autumn for the purpose of verifying some details. My harmless excursion having been alas! indefinitely postponed, I gladly avail myself of the hospitality offered by the Journal of Theological Studies in respect of a few glosses which have a theological or philosophical content, and which exhibit a ninth-century commentator bent on edification.

A brief notice of the man and his activities may be of interest.

Remigius ${ }^{1}$ was probably a Burgundian and a relative of Lupus of Ferrières, and certainly the pupil of Heiric in the Benedictine house of St Germain at Auxerre. He was born not long after his master, about $84 \mathrm{I}$, and succeeded him as head of the school. He did so well and won such fame that he was presently summoned to Reims by Archbishop Fulco for the purpose of building up the double school there (canonicorum scilicet loci atque ruralium clericorum) after the Norman devastation. When Fulco was assassinated in goo by Baldwin,

1 This account of Remigius is founded upon Hist. litt. de La France $\mathrm{t}$. vi ; Haureau Histoir de la phil. scolastique t. i pp. Ig9 ff; the same writer's article 'Remi d'Auxerre' in Didot's Biographie generale; Manitius Geschichte der latrinischen Lilt. des Mittelalters pp. $50_{4}$ ff. 
Count of Flanders, Remigius removed to Paris, where he opened a school, probably connected with the Monastery of Saint-Germain-desPrés, which the authors of the Histoire litteraire de la France do not hesitate to call the germ of the University of Paris. It was not that. Dr Rashdall has shewn (Univ. of Europe vol. i p. 277 ff) that the University grew out of the Cathedral schools which did not rise to prominence before the end of the eleventh century. But if we may not claim University standing for the School of Remigius, it is at least memorable as the first Public School at Paris known to history, and Remigius is the first master of note in the city which came to be known as the nurse of letters.

We know something of the programme of studies there from the Life of Odo, afterwards Abbot of Cluny, who, like Hildebold of St Mihiel, the grammarian, and Blidulf, archdeacon of Metz, sat at the feet of Remigius. His diebus (Odo) adiit Parisiss ibique dialecticam Sancti Augustini Deodato filio suo missam ${ }^{1}$ perlegit et Marcianum in liberalibus artibus frequenter lectitauit. Praeceptorem quippe in his omnibus habuit Remigium (see Migne $P . L$. I 33 col. 62). The matter and method of Remigius's instruction may be gathered from the glosses printed below, at least so far as concerns the interpretation of a Christian text ; for the men of the ninth century, with surer instinct than those of the nineteenth, regarded Boethius as a Christian and a martyr.' In the same category as the Boethius may be placed the commentary on Sedulius, of which extracts are given by Huemer in the appendix to his edition (Vienna Corpus vol. $\mathrm{x}$ ). Remigius also busied himself both with theology proper, and with authors whom no amount of gymnastic could render other than secular. Thus he certainly expounded Genesis and the Psalms (Migne P.L. I $3 \mathrm{r}$ ), probably also the Song of Songs, and possibly other books of the Bible $^{3}$; while the liberal arts which are handled with affection whenever Boethius, Sedulius, or Martianus Capella provide occasion, would claim full play in the commentaries on Donatus, Priscian, Phocas, Eutyches, Cato Minor, Juvenal, Persius, Terence, and the Ars metrica of Bede. All of these are attributed to our author; but the only one which has, so far as I am aware, been printed in entirety is the Donatus Minor, edited by W. Fox for Teubner's Series (1902). Twelve of Remigius's Homilies are given by Migne, and his work on the Mass, to which ancient catalogues of MSS frequently

\footnotetext{
1 i. e. the spurious Categoriae decm, which is chiefly interesting as supplying the theme of Alcuin's verses addressed to Charles the Great, Contimet istedecm naturae uerba libellus, \&c., ap. Aug. Op. (ed. Gaume) I col. I 329.

2 It is true that this instinct leads Remigius to read into the De cons. phil. more explicit Christianity than it really contains.

3 Cf. Manitius op. cit. Pp. 5 I6 ff.
} 
refer, is probably that which figures as ch. 40 of pseudo-Alcuin's $D e$ diuinis officiis.

Of Remigius outside his school and his commentaries we know practically nothing. He is said to have retired to Lorraine where he died in the first decade of the tenth century. His obit in the Auxerre Necrology (May 2) calls him Monachus et egregius doctor. To this we may add that in regard to the 'scholastic problem' he was an extreme Realist, going back to John the Scot across his own master Heiric, who had adopted Nominalism. In realizing abstractions, indeed, he outPlato's Plato. The commentary on Cons. iii m. 9 (see below) proves his intimate acquaintance with the Timaeus, which Boethius had in that metrum versified, but it contains nothing so pronounced as the passage from his commentary on Martianus Capella, where $d$ propos of Celatam sphaeram, he says: Per sphaeram uult intelligi mundum inuisibilem qui in mente Dei latebat antequam iste uisibilis per uarias perduceretur causas; quem mundum, id est inuisibilem, philosophi uocant ideas, id est formas. ${ }^{1}$ There is thus no doubt of the character of the philosophy professed by this first teacher of dialectic at Paris.

Hard things have been said of Remi as a commentator, ${ }^{2}$ and it is true that few of his remarks are likely to make the pulse beat quicker. But it must be remembered that they are but lecture notes, from which originality is not necessarily or as a rule expected. To settle the sources whence they are drawn is a matter of great delicacy. It is quite certain that he used the notes of Heiric, as Heiric had used those of John the Scot; it is possible that his adventures in Greek (of which he has no reason to be proud) are not his own. But whether forged on his own anvil or taken ready-made from elsewhere, the comments are, I believe, of value as exemplifying the educational methods of the early Middle Ages.

Note. $K=$ Maihingen I 2 lat. ( $\mathrm{x}-\mathrm{xi}$ cent.).

$L=$ Trèves I093 (xi cent.).

$C=$ Trin. Coll. Camb. o. 3. 7. (xi cent.).

$F=$ MS belonging to $\mathrm{S}$. $\mathrm{C}$. Cockerell, Esq. (x-xi cent.).

Words enclosed in ( ) are wanting in $K$.

$", \quad "\langle\rangle, \quad, \quad, L$.

I have in the text used the ordinary orthography, and I have not encumbered my apparatus criticus with all the wrong spellings of $K$. The lemmata are quoted from $\mathrm{R}$. Peiper's edition of the De cons. phil. in the Teubner Text Series.

1 Quoted by Haureau Hist. de la phil. scol. t. i 205.

${ }^{8}$ e.g. 'Je mehr Werke des Remigius wir finden, um so geringer erscheint die Originalitat seines Geistes.' Rand op. cit. p. 96. 
I pr. I (Peiper, pp. 4, 5). Supra uerticem. Quia ibi sedes est sapientiae. Mulier. (Ideo) Boetius in specie mulieris philosophiam sibi uisam esse dixit, quia et apud Graecos et apud Latinos feminini generis pronuntiatur, haec philosophia haec sapientia ; uel ideo quia auditores suos quasi quibusdam rudimentis ad perfectam scientiam adducit ueluti mater lacte teneros lactat et nutrit a. Venerandi uultus dicitur esse quia philosophia quos repleuerit reuerentia dignos facit. Ultra hominum ualentiam .i. possibilitatem (quia nullus potest naturam dei comprehendere). Perspicacibus autem .i. ualde aspicientibus, acutis, lucidis, quia nihil perspicacius est sapientia. Pulsare caelum dicit propter astrologiam. Philosopbia duobus modis pulsare caelum dicitur cacumine sui uerticis, cum sublimioribus et perfectioribus sublimiora loquitur et disputat, uel tunc cum de diuinis disserit totidem modis. Et sese ad mensuram hominum cohibere dicitur scil. cum modicis condescendit intellectibus uel cum de temporalibus disputat, altius autem caput extollere, propter theologiam .i. propter diuinam rationem.

(Vestes liberales artes uel doctrinae ingenia uel diuersae disciplinae.) Vestes philosophiae tenuissimis filis perfectae esse dicuntur, quia vil liberales artes adeo subtilissimae sunt ut nec origo earum nec finis aduerti possit. Unde et a Graecis cyclicae ${ }^{\mathbf{b}}{ }^{1}$ dicuntur eo quod origo illarum sicut et circuli omni modo lateat, eoque ipsae artes indissolubiles materiae sunt (ut etiam) desint qui eas capiant ; ipsae tamen nullo modo pereunt, licet enim desit scientia, scibile tamen semper erit.

Caligo quaedam. Caligo quae obduxerat uestes philosophiae errores intelleguntur philosophorum, quia in multis locis contraria dixerunt, uel quia neglegentia in artibus accesserat. Ab inferiori scil. elemento .i. II ad superius elementum .i. litteram $\Theta$, quia per actiuam peruenitur ad contemplatiuam, nam ab actuali scandendum est ad uitam speculatiuam et a terrenis ad caelestia.

Vestem Violentorum sciderant manus .i. Sensus uim inferentium haereticorum praue intellegentium qui scindunt sanam doctrinam suis baeresibus. Haereticos dicit qui nequaquam perfectam sapientian sed quandam partem sapientiae adepti sunt. Unde et suis sectatoribus ex suis nominibus uocabula dederunt, ut alii dicerentur platonici, alii epicurei.

Libellos. Libelli quos in manu dextera gestabat ipsi sunt in quibus liberales artes continentur. Unde et legitur : In dextera eius ignea lex. ${ }^{2}$ Aut libellos disertam doctrinam uel theoricam uitam accipimus ${ }^{\circ}$. Per sceptrum mundana gloria intellegitur quae per sapientiam regitur ${ }^{d}$. In

\footnotetext{
1 Cf. Mart. Capella ix 998.

3 Deut. xxxiii 3.

- lacte nutrit filios $L \quad$ b ciclyce $L$ : ciclide $K \quad{ }^{\circ}$ accipitur $L$ : insignificiant $K$ d quaeque sapientia legitur $K$
} 
sinistra tenetur quia: In sinistra illius diuitiae et gloria. ${ }^{1}$ Sceptrum namque primum uirga est, deinde inauratur et per disciplinam peruenitur ad gloriam.

I pr. 3 (p. 8, 1. I 8). Praeceptor eius Sacrates. In Socrate a magistro Platonis magnum certamen sustinuit philosophia, quia ${ }^{b}$ cicutam compulsus est bibere, herbam ueneniferam, eo quod nollet iurare per deos, per Iouem uid. et per Apollinem et alios. Iurabat autem per lignum, per petram et per similia, dicens deos nihil esse, lapides ${ }^{\circ}$ uero esse. Passus est hic ab Atheniensibus sicut beatus Augustinus dicit, ${ }^{2}$ unde et patientiae famam apud philosophos promeruitd.

Epicureum uulgrus (1. 20). Philosophicum. Physici. Epicurei summum bonum dicunt uoluptatem e, et animam perire cum corpore; Stoici autem dicunt uniforme esse $\mathrm{f}$ omne peccatum, qui dicti sunt a stoa i. porticu.

Abreptis ...panniculis (1. 24). Partibus de haereticis dicit, quia quidam sapientiae assecuti plenam in se putauerunt accepisse sapientiam cum ipsi, sicut hic loquitur, non nisi panniculos ${ }^{8}$ assumpserunt. Unde ab improbis uere sapientes arbitrati pro sapientia sunt damnati. Nulla est enim baeresis quae non habeat aliquid ueritatis. Panniculos autem sententiolas aut singulas artes uix habentes, cum integritas ${ }^{\text {h }}$ philosophiae in septem artibus constet.

Anaxagorae fugam etc. (1. 28). Nomina Graecorum philosophorum. Anaxagoras philosophus propter sapientiam fugatus est a patria et diu exulatus.

I m. 4 (p. ro, 1. 5). Minaeque ponti etc. Sb. mouebunt (a stabilitate mentis). Per pontum .i. mare designat mundum, populorum commotionem per rabiem ponti. Per montem ignem ${ }^{i}$ exhalantem principum comminationem; per uiam fulminis soliti ferire turres, iram regum, fulmen enim turres euertere solet et reges sublimioribus indignantur. Quicumque ergo subiicit sub pedibus suis superbum illud fatum, ille et popularem commotionem ${ }^{j}$ et comminationem principum et minas contemnere potest.

Aestum (1. 6). Commotionem uel ebullitionem. Per commotionem ponti significat perturbationem saeculi in monte superbi atque elati. Intellegitur in fulmine regia potestas ${ }^{\mathbf{k}}$ quae turres .i. potentes saeculi deicere solet.

I pr. 4 (p. 15, 1. 133). Ut consimilem deo faceres .i. Rationabilem, non aequalem ${ }^{1}$.i. secundum iustum ${ }^{\mathrm{m}}$ ad cuius imaginem conditi sumus.

\footnotetext{
1 Prou. iii 16.

De Cir. Dei viii 3.

- In Socr. uid. $L$ b phil. qua $L$

a meruit $L$ dicuntur uoluntatem $K$

in pann. $K \quad$ integritate $K$

- lapidibus $K$ : lap. autem $L$

k intelleguntur regina potestasque $K \quad{ }^{1}$ rationalem non equidem $K \quad$ m $\operatorname{secm}$ et iustum $K$
} 
Et imago quidem dei nobis est in intellectu et mentis rationabilitate. Similitudo est in morum probitate et animae puritate.

I m. v (p. 16, l. 2). Perpetuo nixus solio. Non transitoria residens potestate. Deum ergo inmutabilem in se esse ostendit quamuis cuncta uerset.

I pr. v (p. 19, l. 14). An ignoras illam ... legem. Hoc tam ad Rómae ciuitatem quam ad ecclesiam seu ad caelestem Hierusalem referri potest. Siquidem olim lex romanis sancita est ut quicumque domum habitandi Romae fundasset iam non esset ei fas usquam exsulare, quippe cum totus orbis romanus esset et ubique essent ciues romani. Sic ergo quisquis ecclesiam dei per consortium adierit ab ea peregrinari non debet. Nemo, inquit, mittens manum suam in aratrum a et respiciens retro aptus est regno dei, ${ }^{1}$ seu etiam quicumque supernorum ciuium societatem et sedem in illa superna ciuitate semel exceperit numquam ei fas esse potuerit exsulare a deo, quia erit deus omnia in omnibus.

Tuae ciuitatis (ib.). Supernae scil. Hierusalem.

I m. vi (p. 20, l. I5). Backzs. Poetice deum posuit.

I pr. vi (p. 21, l. 7). Rationis. Diuinae dispositionis. Ratio dei alia est et alia hominis. Ratio hominis est discernere inter bonum et uerum, ratio (uero) est aeterna dispositio.

ib. (1.8). Certa. Neutraliter posuit ${ }^{\mathrm{b}}$, ea uid. quae a uero deo in ueritate sunt facta non fortuitis casibus disponuntur sed creatoris moderatione.

ib. (1. 9). Praesidere. Praesidere est auxiliando praeesse. Unde praesidere dicitur deus omnibus creaturis gubernando et regendo. Hac etiam ratione semper operari dicitur, non noua creando sed gubernando creata.

ib. (1. 23). Quid sit rerum finis .i. deus, finis enim omnium rerum deus est. Corpora enim in quattuor elementis resoluuntur ex quibus constant. Rursus elementa in ylen .i. informem materiam. Yle enim ad deum revertitur ${ }^{\circ}$ quod utique (a deo) factum est. Necesse est enim ${ }^{d}$ ut unde originem sumant ibi separentur. Omnis quidem creatura a deo originem capit et in eo cuncta resoluuntur.

I m. vii (p. 23, 1. 25). Gaudia pelle. Quattuor animae notissimas passiones bic tangit, quarum duae ab opinatis bonis sunt, hoc est et gaudium et spes, et una de praesenti, altera est de futuro, gaudium de praesenti, spes de futuro. Duae etiam ab opinatis malis, quarum una est de praesenti et altera de futuro similiter, dolor sc. de praesenti, timor de futuro. Quae etiam Virgilius commemorat : Hinc cupiunt, metuunt, gaudentque dolentque." Nam spes et cupiditas idem. Dicit

\footnotetext{
1 Luke ix 62 .

- in aratrum et cętera $K$

d est autem $L$
}

\footnotetext{
b posuit certa cuncta $K$

- uertitur $K$
} 
ergo quicumque his subiacent passionibus animum liberum et rationis plenum procul dubio se non habere demonstrant. Nam et Cicero dicit quia si iudex has tulerit passiones uerum fari non poterit.

II m. iv (p. 35, l. 92). Quod si multos etc. Hic sanctos martyres uult intellegi qui ut beatitudinem per hominem acciperent, diuersa potius tormenta quam simplicem mortem desiderabant.

II m. 8 (p. 49, 1. 15). Caelo imperitans amor. Amorem uocat deum quia elementa uel tempora cum inter se discordare uideantur, in eo tamen concordant et ad-eum (cuncta) quasi ad certun finem contendunt; non ad eius quidem naturam sed ad id quod dicitur omne quod est deus qui omnia fecit et omnia regit.

ib. (1. 18). Bellum continuo geret. Faciat. Si deus (inquit) sua uirtute mundum disponendo non regeret, omnia essent illico confusa. Ideo ait in euangelio (dominus noster Iesus Christus): Pater meus usque modo operatur et ego operor ${ }^{1}$; non quia noua condat sed quod condita seruet ${ }_{2}^{\mathrm{a}}$. Ita ergo deus semper operari dicitur.

III pr. 2 (p. 52, 1. 13). Veri boni cupiditas. Quia bonae sunt creatae ad similitudinem (dei).

ib. (1. 25). Felicissimum putant uoluptate diffuere. Secundum Epicurum qui summum bonum dicit esse. (Sunt qui) uoluptatem adhuc uoluerunt diuitiis abundare ut uoluptuose uiuant sicut Epicurei et eneruati.

III m. 2 (p. 54, l. I). Quantas rerum flectat kabenas etc. Thema hoc sumptum est ex eo ubi paulo supra dictum est (quod mentibus hominum naturaliter inserta est ueri boni cupiditas), in quo quanta sit naturae uis facile monstratur. Ostendit autem omnem rem suam retinere naturam et licet aliquid deuiet $\langle$,ad eandem tamen redire conatur $\rangle$. Demonstrat etiam omnia essentialiter esse bona, quia deus bonus omnia bona creauit. Non tamen iusta sunt (omnia) quae sunt bona. Bona enim in essentia et natura, iusta uero sunt in actu et opere. Hinc et diabolus bonus dicitur essentialiter, non tamen iustus 〈est $\rangle$ in opere.?

III pr. I (p. 50, 1. I3). Degustata mordeant. Sapientia dum primum sensibus percipitur dura uidetur. Unde legitur: Verba sapientiae quasi stimuli et claui in altum defixi." Cum uero arcano corde perpenditur dulcescit. Unde est : De ore prudentis procedit mel," quia uid. cum uerba sapientiae utiliter meditantur, dulcedinem quasi mellis tribuunt menti.

III m. 6 (p. 63, 1. 6). Mortales igitur cunctos edit nobile germen. Quia a deo omnes immortales et rationales conditi sunt, et plures quidem elephantos multos(que) leones et de aliis animalibus deus

\footnotetext{
1 John v I7.

- Eacl. xii i I.

2 Cf. Aug. De wera mlig. 26; D. Ciu. Dei xix 13.

- 1 cannot trace this quotation.
}

- seruat $K$ 
plura fecit, ad maiorem uero conciliationem humani generis unum tantum a hominem creauit, de quo mulierem produxit. De quibus duobus omnes sumus nati pariter ${ }^{b}$. Omnes igitur similiter (nobiles sunt homines).

III m. 8 (p. 66, 1. I). Ehert quae miseros etc. Dolet ignorantiam hominum per quam a uero bono deuiant, et eo modo argumentatur quomodo dominus ad Iudaeos ${ }^{\circ}$ dicens: $\mathrm{Si}$ uideritis caelum sero rubere dicitis serenum; si mane, dicitis nubilium erit. Hypocritae, faciem caeli cognoscitis, et aduentum filii hominis ignoratis. ${ }^{3}$ Ita hoc loco dicitur, et reprehenduntur homines quod callent agnoscere bona corporum exteriora et nunc quae res quo loco melius reperiantur, uerum autem bonum ad quod naturaliter tendunt patiantur quasi caeci ignorare.

III pr. 9 (p. $67,1.12)$. Traducit. Transfundit. Tradux proprie est transmissio et transfusio. Unde legitur traducem originalem peccati.

$i b$. (p. 68, 1. 4r). Nomina quidem esse diuersa, nullo modo wero discrepare substantiam. Sicut in deo cognoscitur qui d omnia uirtutum dona in se continens ${ }^{\theta}$ diuersis censetur nominibus, ipsa autem eius substantia una est et in deitate simplex.

III m. 9 (pp. 70, 7I). O qui perpetua mundum ratione gubernas. Rationem dicit sapientiam dei per quem omnia creata sunt et gubernantur. Ipse est enim uerbum dei, filius dei, sermo dei, 〈ratio dei〉 et sapientia. Vel perpetuam dicit rationem aeternam dei dispositionem secundum quam omnia constant et creata sunt. Gubernas. Regis. Terrarum caelique sator. Aut simpliciter per caelum et terram quattuor uult accipi elementa quibus omnia constant, aut per caelum angelos, per terram uero homines qui in ea habitant designat. Sator. Metaphora (est) ab animali ad creatorem. Ab aeuo (.i.) ex quo dixisti : Fiat lux, ${ }^{2}$ quoniam ${ }^{f}$ ex tunc coepit $\mathrm{B}$ reuolutio esse temporis. Nam antea non erat tempus sed aeuum i. perpetuitas quaedam. Aeon enim graeci dicunt perpetuum. ${ }^{3}$ Ire $\langle$. i. $\rangle$ currere uniformiter. Das. Facis ; zeugma ab inferioribus : Da, pater, augustam menti conscendere sedem $\mathbf{b}$. Cuncta moueri i. cuncta quae das facis uel loco uel tempore moueri i. Nam corporalia $j$ omnia loco simul et tempore mouentur, spiritalia uero ut anima tempore tantum, non autem loco mouentur. ${ }^{4}$ Deus uero nec loco nec tempore mouetur, sed omnem locum maiestate sua implet et omnia simul in eo sunt tempora. Quem non externae etc. Quem

1 Luke xii 56.

- Cf. Aug. de Gen. ad litt. viii 39.

'Gen. i 3.

- tantum modo $L$ b aequaliter $L$ - continet $K$ ' qũo $K$ cepit $K$ diately after that on cuncta moueri.
Cf. Isid. Etym. v 38. 4.

- ad eos dicens $K L \quad$ a quia $K$ In $K$ this gloss follows imme1 mouere $K$ I temporalia $L$ 
.i. 〈te〉 creatorem non coegerunt causae extrinsecus tibi accidentes ut fingeres mundum, sed ipsa tua beniuolentia naturaliter tibi insita $b$.

Materiae fluitantis opus. Fluitantem materiam uocat illam informem materiam quae in mente dei in primordio fuit ${ }^{1}$ antequam mundus fieret quando omnia fluitabant, neque adhuc facies terrae uel aeris apparebat. Terra namque cooperta ${ }^{\circ}$ erat aqua licet tenui uel nebula $d$; aeris quoque claritas non apparebat, quia nec erat lux qua inlustraretur e. Siue fluitantem materiam huius mundi uocat creationem quae semper fluit et labitur. Summi forma bona. S. coegit te. Formam uocat filium dei qui est sapientia dei, per quem omnia facta sunt." Unde scriptum est : Qui cum sit splendor gloriae et figura eius ${ }^{3}$.i. dei patris. Vel etiam formam dicit illud exemplar et rationem quae erat in mente dei ${ }^{*}$ ad cuius similitudinem post mundus factus est, et ipsam rationem uocat Plato ideas uel formas. Sicut (enim) artifex arcam facturus prius figuram illius in mente praeuidet ad cuius similitudinem post opus facit, ita deus formam huius mundi semper in ratione sua habuit antequam illum faceret ad eandem similitudinem. Beatus uero Iohannes ipsam rationem et dispositionem dei, quam Plato ideas uocat, uitam nominat. Antequam enim mundus fieret $f$, in mente dei erat, et antequam caelum crearetur in arce uiuebat. Ideo ergo ipsa ratios uita uocatur, quia semper uixere. Lizore carens. Quia non inuidith creaturae suae faciendo eam ad similitudinem suam. Ducis. Próducis. Ab exemplo. Quod dicit formam, hoc exemplum. Mente .i. dispositione tua.

Similique in imagine formans .i. (per) filium qui est imago et similitudo patris. Siue simili imagine .i. sicut in tua dispositione cuncta imaginata erant. Perfectas...partes .i. quattuior elementa quae et perfecti mundi partes sunt et (ipsa) in se perfecta sunt. Perfectum 〈.s.〉 mundum. Absoluere. Explicare, perficere. Ligas .i. coniungis. Numeris .i. quattuor monadibusi, nam quattuor sunt elementa ${ }^{\circ}$ quorum sex sunt connectiones quas (Graeci) synzygias ${ }^{j}$ uocant, $^{7}$ quarum ${ }^{1}$ quattuor sunt immediatae et duae mediatae. Immediatae sunt istae $^{\mathbf{m}}$ : Aer calidus et humidus est ; huius caliditas coniungitur caliditati ignis, qui est calidus et siccus. Ignis est calidus et siccus; huius

1 Wisd. xi $17 . \quad 2$ Cf. Aug. Serm. cxvii 3. 'Heb. i 3.

4 Cf. Aug. De dik. qu. Ixxxiii, xlvi $2 . \quad$ John i 4 .

- Cf. Ambros. 'Unde nec numerum monada sed elementum numeri ... appellauerunt', Ep. vii 22.

${ }_{7}$ Cf. Aristot. De gener. et corrupt. ii 5 ; Meteor. iv 1 ; Bridf. Gloss. on Bede De Nat. rer. iv. Byrthferth of Ramsey (n. 1000) quotes R. twice by name (Migne P. L. $90 \mathrm{col} .3^{\mathrm{IO}}$ ).

- sibi $L \quad$ b indita $K$ : insita non inuidens creaturae ad imaginem et similitudinem tuam formare $L$ o operta $K$ d nubila $K$ illust. $L$ I Nam anteq. f.m. $L$ Ergo ratio illa $L$ ' inuidet $L$ I nomadibus $K$ I el. s. $L \quad$ sinzuglas $K L \quad 1$ quorum $K \quad$ miste $K$ 
caliditas aeris caliditati coniungitur. Siccitas autem terrae copulatur, quae est frigida et sicca. Terra frigida est a et sicca; huius siccitas ignis siccitati coniungitur ${ }^{b}$. Frigiditas uero aquae frigiditati nectitur. Aqua frigida est et humida; eius frigiditas terrae frigiditati (copulatur). Humiditas autem aeris humiditati sociatur. Mediatae synzygiae ${ }^{\mathfrak{c}}$ hae sunt quae contrariae sunt nec possunt coniungi sine aliqua medietate. Ignis et aqua contraria sunt, quia ignis calidus est et siccus, aqua frigida et humida. Nam ut $\mathrm{d}$ frigiditas aquae ignis conueniat caliditati, terrae frigiditas est media ; ut autem aquae humiditas siccitati ignis aptetur, aeris humiditas media interuenit. ${ }^{1}$

Ut frigida e flammis. Terra frigida coniungitur igni ex ea parte qua siccus est. Arida conueniant liquidis. Terra arida coniungitur aquae ex ea parte qua frigida est. 〈Ne prorior ignis euolet. Ignis duobus crassioribus se elementis tenetur, aqua scil. et aere ne ad sedem suam euolet, in aethere enim eius sedes est ; unde semper superiora petit $\rangle.$ Mersas Pessumdatas. Pondera (s.) terrae uel aquarum. Terra ponderata est, unde et omnia quae pondus habent, de quacumque caeli parte cadant, ad terram feruntur ; quia uid. in imo posita 〈est ) undique caelum (sursum) habens $f$ ne subsidat pondus eius (et) ad nihilum deducatur. ${ }^{2}$ Duobus elementis leuioribus sustentatur, aqua uid. et aere. Tu triplicis etc. Vis animae omnem molem corporis regit. Philosophi animam mundi solem dixerunt esse quia sicut calificat corpus anima, ita solis calore uiuificantur omnia, eiusque calor diffusus per creaturas facit eas gignere, et re uera ut physici $\mathbf{B}$ dicunt calore illius omnia et gignunt et gignuntur ${ }^{b}$ pariter cum humore, deo ita disponente. Hic itaque sol triplicis naturae est; habet enim esse, habet calere, habet splendere." Sol enim medius est inter planetas. Primus enim Saturnus est, deinde Iouis, inde Mars ${ }^{1}$. Hi sunt superiores tres, et quartus est sol. ${ }^{\circ}$ Sub eo uero reliqui tres, Venus et Mercurius et luna.

Mediam animam. Media dicitur anima non quod a meditullio corporis i. ab umbilico sit porrecta, sed quia ${ }^{j}$ in corde sedes illius proprie est $k$ ubi pontificium 1 est uitae." Aut certe media dicitur quod est anima rationabilis media inter animam pecudum et spiritum angelorum. Omnis namque $m$ spiritus aut cum carne tegitur et cum carne moritur, aut nec carne (quidem) tegitur sed cum carne non

1 Macrob. Comm. i 6.

- Cf. Macrob. Comm. i 22.1 .

- Cf. Macrob. Comm. i 20.6 ; Sat. i 18 . I5.

- Cf. Aug. Sal. i $15 . \quad$ Cf. Bridf. Gloss. on Bede De Nat, rer. xiii.

- Cf. Chalcid. Comm. xcix.

t. egt fr. $L$ b sicc. iungitur $L$ o sinz. $K$ : synzug. $L$ d aut $K$ - ut frigora $K$ ' habet et $L$ o philosophie $L$ b gignit et gignitur $K$ I Mars habens sup. tres $L$ J si quia $K \quad$ \& est proprie $L \quad 1$ pontificum $K \quad$ m omnis autem $K$ 
moritur, aut nec came tegitur nec (cum carne) moritur. (Anima pecudum carne tegitur et cum carne moritur. Anima hominum carne quidem tegitur sed cum carne non moritur), spiritus angelorum nec carne tegitur nec moritur a. Prudentioribus autem uidetur hoc loco potius animam rationabilem debere intellegi quae magnam concordiam habet cum mundo $b$. Unde et homo graece microcosmus dicitur .i. minor mundus. ${ }^{1}$ Sicut enim mundus c quattuor elementis et quattuor temporibus constat, ita et homo quattuor humoribus et quattuor flatibus. Videamus ${ }^{d}$ ergo (mundi) et hominis concordiam. Quattuor sunt elementa : aer ignis aqua terra. Aer calidus et humidus est ; uer calidum et humidum similiter. ${ }^{3}$ (Et) sanguis 〈qui〉 est in puero aeque calidus et humidus ${ }^{\ominus}$; pueritia (enim) calida et humida (est). Ignis calidus est et siccus ; restas calida et sicca. Colera rubea quae abundat in adolescente calida et sicca; adolescentia enim calida et sicca. Terra frigida et sicca; autumnus frigidus et siccus. Melancolia $f$.i. colera nigra quae est in iuuenibus frigida et sicca; iuuentus (enim) frigida et sicca. Aqua frigida et humida est; hiems frigida et humida. . Flegma quae abundat in senibus frigida et humida; senectus frigida et humida. Iste ergo (minor) mundus habet animam triplicis naturae ; est enim irascibilis concupiscibilis rationabilis. Irascibilis, ut uitiis irascatur et corporis uoluptatibus; concupiscibilis est ut deum diligat et uirtutes appetat ; rationabilis est ut inter creatorem et creaturam, inter bonum et malum discernere possit. 〈Quae tria si rationabiliter fuerint custodita coniungunt creaturam creatori.) Si uero fuerint permutata, mentem debilem reddunt, (nam) si illa pars fuerit corrupta quae irascibilis dicitur, fit homo tristis rancidus felle amaritudinis plenus. Si autem illa pars fuerit uitiata $\mathrm{g}$ quae concupiscibilis dicitur, fit homo ebriosus libidinosus et uoluptatum seruus. Si uero illa pars animae corrumpatur ${ }^{\mathrm{h}}$ quae uocatur rationabilis, fit homo superbus, haereticus, omnibus subiectus uitiis $\mathbf{i}$.

Per consona membra. Armonica enim (disciplina) corpus humanum compositum est. Hinc Sedulius : Et reuocata suis attemperat organa

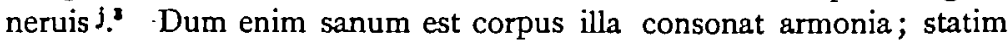
autem ut dissentit aegrotat corpus. Resoluis (s.) inmittis, infundis: Quae cum secta dwos motum glomerauit in orbes. Non est anima in sua * natura diuisa, sed actus ipsius in duos extenditur oculos ad aliquid contemplandum, sicque dicitur glomerare suum motum in duos orbes et reuertitur in semet ipsam. Dicunt (enim) doctores quod per intuitum

1 Cf. Chalcid. Comm. cc; Arnob. Adv. Nat. ii 25.

2 Cf. Macrob. Comm, i 6.60; Sat. vii 5.20 f.

- nec carne m. $L \quad$ b concordiam inter cum m. $K$

a uide'mus $L$

3arm. paschale 3,256. c minor mundus $K$ fu $L$ helancolica $K$ uic. bris $K:$ o.m. neruis $L \quad$ sui $L$

VOL. XVII. 
oculorum uis animae egreditur ad conspiciendum exteriora, ita tamen extendit se a ${ }^{\text {a }}$ utatim reuertatur per profundam meditationem in se reuoluens agensque (in) simili imagine quae foras uidet. Sicut (enim) uidet $b$ exterius uoluit. Ita est de aliis rebus intellegendum quae prius uidit et deinde meditatur. Nam cum unus sit sol, radios in diuersam partem ${ }^{\circ}$ uidetur diuidere, cum per rimulas et fenestras ingreditur (In duos orbes) .i. in ortum et occasum. Unde Salomond dicit : Oritur sol et occidit et reuertitur ad ortum suum; girat per meridiem et flectitur ad aquilonem. ${ }^{1}$ In semet reditura meat meniemque profundam circuit .i. aeternam dei et profundam dispositionem peragit. Simili conkertit imagine caelum .i. aequali modo, simili semper cursu. Vel simili imagine ut subaudiatur qualis fuit illa in qua ${ }^{\circ}$ creatus est, uid. in aequinoctiali et occasu.

Conuertit i. conuerti facit, nam dicunt quod impetu solis uoluentis contra mundum retineatur sphaera $f$ caelestis semper uoluens ne labatur et pessum ruat. Tu causis etc. Oṛdo uerborum est : Tu prouehis animas uitasque minores paribus causis, et seris in caelum et terram aptans sublimes animas leuibus curribus. Diuerso modo diuersi in hoc sentiunt. Quidam (enim) ita intellegunt ut animos dicat angelicos spiritus, uitas uero minores homines quos paribus causis produxit, dum eos rationabiles condidit seritque .i. inmittit $\mathbf{s}$ angelos in caelum, homines in terram aptans sublimes animas leuioribus curribus .i. subtili contemplatione $^{\mathbf{b}}$ ad consideranda caelestia. Alii animas doctos et sapientes intellegunt, uitas minores stultos, ut serat in caelum sapientes, in terram stultos, et sublimes animas sapientium leuioribus curribus aptet .i. subtili intellectui i. Attamen prudentioribus aliter uidetur. Animas rationales hominum spiritus intellegunt, uitas uero minores pecudum animas. Duae enim $\mathbf{j}$ sunt, rationalis quae est hominum, et uitalis quae est animalium. Unde quia tantum ad usum uitae animam habent, graece $z \omega^{k}$ dicuntur $^{1}\langle$, Zoe enim graece uita dicitur $\rangle$. Hinc quidam uolunt Zodiacum illum (circulum) signiferum quod animalia habeat, taurum leonem et reliqua.

Prouehit ergo deus animas et uitas paribus causis .i. aequali potentia, et hominem quidem ad imaginem et similitudinem suam condidit et animalibus uitalem tribuit animam $m$. Has ergo animas sublimes rationabilitate aptat leuibus curribus .i. subtili contemplationi et intellegentiae, easque serit in caelum .i. ad caelestem instruit ${ }^{\mathbf{n}}$ conseruationem. Vitas uero minores serit in terram, quia animalia tantum terris dedita

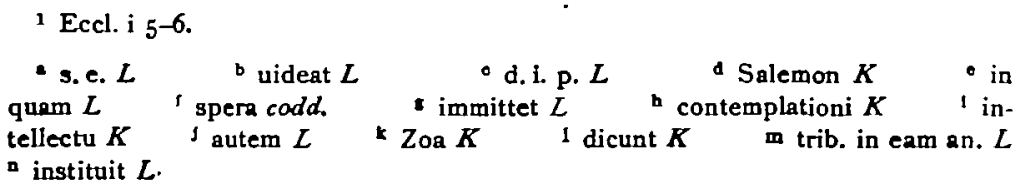


sunt et cum moriuntur corpore moriuntur et anima. Tu namque serenum .i. serenitas. Tu requies ... te cernere finis. Definitio ast summi boni. Finis et perfectio idem est, sed finis aliquando deest sumptione, aliquando dicitur de consumptione. Unde aliter dicimus finiri telam, aliter panem; panis quidem finitur ut non sit, tela autem ut perfecta sit. Terminus .i. per quem terminum perueniamus ad te.

III pr. Io (p. 72, 1. 3). Quonam haec felicitatis perfectio constituta sit sb. loco: deus namque, si dici fas est, locus est omnium bonorum. Sciendum uero deo non esse aliud bonum et aliud esse ipsum deum, sed ipsum bonum deum esse. Neque enim in eo ita est beatitudo quomodo in homine sapientia et in uase aqua, sed cuncta quae de deo dici possunt, secundum eius naturam et substantiam dicuntur, et ideo a quibusdam dicitur non esse bonus et sapiens quia ipse (est) bonitas et sapientia.

ib. (p. 76, 1. 135). Dei in ipso bono .. substantiam. Locus dei, si dici fas est, bonum et ipsum bonum in deo. Unde potest illa quaestio. solui qua quaeritur ubi erat deus antequam mundum crearet. Hoc est in bono et ipsum bonum deus. ${ }^{1}$

III pr. I 2 (p. 82, 1. 23). Hoc quicquid est etc. Naturaliter dicit hoc b i. illa substantia quaecumque est, et ideo quasi cum quadam dubitationedicit hoc quicquid est, quia deus inuisibilis est omnibus rebus, neque enim ipsi àngeli integre ualent considerare eum, de quibus scriptum est : In quem desiderant angeli prospicere. ${ }^{2}$ Sciendum tamen quia secundum aliquid semper uidetur $a b$ angelis. Hinc legimus: Quia angeli eorum semper uident faciem patris mei qui in caelis est"; et cum idem deus ubique summe sit, nihil tamen esse dicitur quod eius ignoratur. Similiter cum sit ipse lux et habitet lucem inaccessibilem, tenebrae asseritur esse propter considerantium infirmitatem. ${ }^{\circ}$

ib. (p. $83,1.52$ ). Naturam seruans. Bene dicit naturam seruans quia in quantum naturam seruat qui bene agit et a deo regitur, in quantum autem a deo discedit male agit et contra naturam fertur. Nam et diabolus in sua natura bonus est et potestas eius quidem iusta,

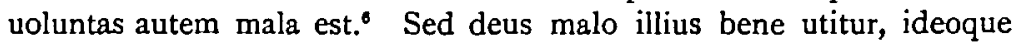
dum aliquid conatur malum, uoluntate tantum deo resistit, ceterum quicquid agitur deo oboedit inuitus, quoniam quod deus permittit fieri iusto iudicio hoc tantum ille agere potest.

1 Cf. Aug. Enarr. in Ps. cxxii 4 .

Matt xviii 1o. Cf. Aug. Contra Max. ii 9.

2 I Pet, i i 2.

4 I Tim. vi 16.

s Cf. Iohannes Scottus De dixis. nat., M. P. L. 22 col. 681 B 'Proinde a theologia caelestium uirtutum claritas saepe nominatur tenebrositas. Nec mirum, cum et ipsa summa sapientia, cui appropinquant saepissime tenebrarum uocabulo significetur. Audi Psalmistam : quia sicut tenebrae, its et lumen eius.'

- See above.

diff. $L$

' hec... hęc $K$ 
IV pr. I (p. 89, 1. 16). In regno scientis omnia .i. in hoc mundo; licet enim in hoc mundo saepius mali praeualent, tamen regnum dei bene dicitur quia a deo gubernatur et regitur; uel in regno dei, hoc est in ecclesia dei quae adhuc est partim in mundo.

ib. (1. 21 ). Dispositissima domo uilia wasa etc. (.i.) in ecclesia uel in mundo. Hoc ex Paulo apostolo sumptum est qui in una domob quaedam uasa esse dicit in honorem, alia (uero) in contumeliam. ${ }^{1}$

IV m. I (p. 89, 1. I). Pennae .i. alae contemplationis. Hae sunt illae alae quas habebat illa mulier quam uidit Iohannes in apocalypsi amictam sole et luna sub pedibus eius.

ib. (p. 90, 1. 17). Aetheris. Possumus quoque per terras nubem aerem et cetera hominen, per aetherem uero astra planetas et reliqua: caelestia angelicos ${ }^{\circ}$ spiritus intellegere.

ib. (p. 94, l. 99). Sed eosdem esse ... nego. Hoc non de quolibet malo dicendum qui utique conuerti potest, sed de diabolo principe malorum, qui angelus a deo factus est, sed quia ex suo malo malus est iam non angelus simpliciter sed cum additamento angelus malus uocatur."

IV pr. 4 (p. 102, 1. 73). Alia uero purgatoria clementia etc. Quia dixit miseriores esse reprobos sua improbitate quoniam perpetua eorum miseria efficitur, nunc iam transit ad nostrum dogma quod docet animas post mortem supplicia luere uel ut purgentur (uel) ut perpetuo damnentur. Igni enim corporeo cruciantur quamuis incorporeae sint. ${ }^{\mathrm{d}}$

IV pr. 5 (p. 106, 1. 23): Sed tu quamuis causam tantae dispositionis ignores. Cum enim ${ }^{\theta}$ cernimus bonos in conualle huius saeculi positos aliquando prosperari aliquando contraria pati, recte fieri omnia credere debemus quia iudicium dei occultum est sed semper iustum et non semper occultum $\mathrm{f}$.

IV pr. 6 (p. io8, 1. I I). De prouidentiae simplicitate, de fati serie. Inter prouidentiam et fatum hoc distat: prouidentia est occulta dei dispositio in intimo diuinae mentis latens quae simul cuncta complectitur qua disponit (deus cuncta quomodo stant. Sicut artifex fabricationes aliquid omnia prius per artem quam habet in mente disponit) et sic post operatur. Fatum uero est cum paulatim foras prodire et manifestare coeperit in rebus exterioribus. Veluti opus in mente artificis comprehensum prius quod postea perficitur exterius. Sed beatus Gregorius dicit nihil esse fatum. Sed si fatum aliquid est dicendum, fatum est naturalis ordo rerum ex prouidentia dei uenientium. Siquidem fatum dicitur locutum et dixisse dei fecisse est, dixit enim $B$ et facta

\footnotetext{
12 Tim. ii 20.

- Greg. M. Hom. $x$ in Euang. 4. Apoc xii I.

3. Ixxviii 49.

- secula $K$ b ditissima domo $K$ o angelorum $L$ a corpore et sint $K$ - autem $L \quad$ f oce. est sed semper est occ. $K$ autem $L$
} 
sunt, mandauit et creata sunt. ${ }^{1}$ Fatum igitur est opus dei quod ex prouidentia descendit.

ib. (p. 108, 1. 1 2). De arbitrii libertate quaeri solet. uid. quod praedestinatione dei sit in electos eius et quod liberum arbitrium habent ipsi. Si igitur ad praedestinationem uenire coguntur, quomodo est eis liberum arbitrium, et rursus ${ }^{b}$ si liberum habent arbitrium, quomodo praedestinationi subiacent?

ib. (p. 108, 1, 22). Quicquid aliquo mouetur modo .i. loco uel tempore. Nam corporalia in loco simul mouentur et tempore localiter, incorporalia uero in loco sunt et in tempore mouentur, sed inlocaliter, et quia de corporalibus patet de incorporalibus dicendum est aliquid. Anima siue angelicus spiritus in loco est. Nam si ubique esset, deus esset (in loco). Ergo in loco quidem est quia non potest ubique esse, sed inlocaliter. Si enim localiter esset, necessario sex loci partes haberet, ante post dextram sinistram super infra. Sed haec minime habebit quia incorporalis est. Si igitur in loco est inlocaliter, inlocalis utique est quia loco minime mouetur. Solummodo ergo tempore mouetur quod in reminiscendo quae non nouit et obliuiscendo eorum quae nouit dicitur 0 constare.

ib. (p. 108, 1. 32). Fatum wero \&c. Nota fatum nihil esse, ut beatus Augustinus ${ }^{2}$ aliique dicunt, sed quod prouidentia dei disponente foris agitur fatum uocatur.

ib. (p. I08, 1. 34). Prowidentia namque cuncta \&c. Illud quod in mente creatoris disponitur atque ordinatur qualiter proficiatur, prouidentia est. Dum uero id ipsum sensim per genera et species foris perficitur, fatum uocatur eo quod non simul procedat sicut simul dispositum est, ac per hoc quasi uariabile et mobile est fatum.

ib. (p. Iog, 1. 50). Sew caelestibus siderum motibus. Hoc in loco quidam conati sunt Boetium reprehendere quasi haereticum, dicentes illum mathematicam sectari et docere omnia sub d fato stellarum fieri. Sed sciendum est quia ubi plura succincte et comprehensiue memorantur ignoratur quod debeat eligi, quod eligendum uel quod respuendum ${ }^{\theta}$ sit.

ib. (p. 1 10, 1. 71). Ac tanto aliquid fato liberum est quantum illum rerum cardinem uicinius petit. Nam sancti aliquando in bac fragili carne positi fati seriem transcendunt ad tempus. Beatissimus enim Benedictus noster pater ${ }^{3}$ quando simul totum mundum conspexit diuino spiritu ultra omnem rerum naturam subleuatus est. Tunc ergo et fati seriem transcendit. Sed mox quia mortali erat carne circumdatus cognouit se intra corporis saepta deteneri et mortalem esse, aliis hominibus similem. Per sanctitatem igitur quia deo erat proximus fatum excessit, sed rursus per carnem quae erat mortalis ad ipsum relapsus est.

\footnotetext{
1 Pr. xuxii 9. $\quad$ Cf. De Cir. Daiv $8 . \quad$ Greg. M. Dial. ii 35.

- Si ergo $L$ bursum $K$ • dicunt $K$ व $\operatorname{coram} L$ • respondendum $K$
} 
ib. (p. I 1 2, 1. 131). Sapiens dispensatio. Huius rei exemplum in beati Iuliani passione habemus, qui cum traheretur per plateas nobilis puer eiusdem iudicis filius diuino instinctu (in) Christum credens martyrem dei secutus est et in carcerem cum eo missus. Petens ergo apud patrem ut triduo cum matre loqueretur, eam conuertit a ad deum. Interfecto itaque puero cum matrem quoque iussisset comprehendi si quis manum ad eam extendit, statim arefacta est, siquidem prouidebat deus non posse eam tormenta sufferre. Igitur fusa oratione perrexit in pace ad deum $b$.

ib. (p. II3, 1. 144). Alii plus aequo metuunt. Despiciunt. Plus aequo .i. plus quam necesse sit. Ideoque datur eis tribulatio ut suam fortitudinem agnoscant. Sicut legimus quia ${ }^{\circ}$ cum quidam frater temptationem carnis non ferens uenisset ad quendam senem ut consilium ab eo reciperet $d$, ille respondit, numquam se talia passum. Cumque tristis ille recederet, uenit ad alterum seniorem cui et molestiam sui corporis et praedicti senis uerba rettulit. Qui postquam eum consolatus est, uenit contra praefati senis cellulam flexisque genibus orabat ut aliquid carnalis temptationis illi eueniret. Itaque statim uisibiliter conspexit ignitam diaboli sagittam contra eius cellulam dirigi. Et cum iam frater ille se hac illacque ${ }^{\theta}$ conuerteret penitusque nesciret quid ageret et saeculum uellet repetere, dictum est ei ab illo sene beato ne amplius auderet fratrem despicere etiam in tribulatione positum. Sicque a stimulatione illa sanatum $\mathrm{f}$ eum dimisit.

ib. (1. I48). Quidam suppliciis . . . exemplum . . . praetulerunt .s. sicut sancti martyres.

ib. (p. II4, l. I86). APГAЛEON $\triangle E$ ME TAYTA $\theta E O N \Omega \Sigma$ TIANT' AГOPEYEIN. ${ }^{1}$ Prouerbiale exemplum in graeco positum quod corruptum est. Sed latina interpretatio hoc dicitur habere: Irascentem post deum tanquam (omnia conuocare) .i. qui irascitur et aliquid conatur agere contra dei dispositionem, etiam in hoc ipso deo seruit eiusque dispositionem quamuis inuitus peragit. Sicut Ioseph fratres uendiderunt ne illorum esset dominus. Verumtamen nisi esset uenditus dominus eorum non esset. Similiter Iudaei cum dominum saluatorem nollent recipere dicentes : Ecce totus mundus post eum abiit $\mathrm{B}$ et nos nihil proficimus ${ }^{\mathrm{h}} \mathbf{9}$ : dumque illum occiderent, uolentes nomen eius extinguere, amplius illud dilatauerunt et dum irati resisterent ipsi i famulati sunt. Neque enim totus mundus in Christum crederet nisi Iudaei eum interfecissent.

IV pr. 7 (p. II8, 1. 45). Proelium . . conseritis. Ut beatus Augustinus aliique sancti doctores dicunt, non magis est fortitudo necessaria in aduersis quam in prosperis; aduersitas enim $\mathbf{j}$ quia non

1 Ibiad xii 176.

- eamque conuerteret $K$

I hac atque illac $K$ I sanum

- autem $L$
I John xii 19.

- quod $L$ acciperet $L$ b perfecimus $K$ i illi $K$ 
potest occulte uenire, ideo totis uiribus ei renititur (, prosperitas autem blandiendo decipit et mulcendo plus saeuit quam occulte).

IV pr. 7 (p. r 18, 1. 47). Medium uiribus occupate .i. aequanimitatem in omnibus seruate, stantes inter aduersa et prospera immobiles: uel medium .i. uirtutum. Nam uirtutes philosophi medias esse dixerunt et inter plus minusue esse locatas. Verbi gratia quattuor (sunt) principes uirtutes, iustitia fortitudo prudentia temperantia. Et iustitia inter pleonexiam et mionexiam collocata est ; fortitudo inter timiditatem et audaciam; prudentia inter calliditatem et hebitudinem ${ }^{b}$; temperantia inter luxuriam eneruatorum et insensibilitatem pecudum.

V pr. I (p. I 22, 1. 23). Nam nihil ex nikilo. Persius in dogmate Epicureorum dicit ex nihilo nihilum posse fieri ita ${ }^{\circ}$ : De nihilo nihilum, in nihilum nil posse reuerti. ${ }^{1}$ Epicurei enim duo dixerunt esse principia, hoc est atomos et inane, ex quibus facta sunt elementa. Et atomos dicebant corpuscula fuisse breuissima quae uix uideri poterant, qualia sunt ea quae per solis radios uidentur discurrere. Inane autem uocabant illud spatium in quo atomi continebantur. Haec ergo duo materiam fuisse rerum creatarum d uolebant. Nos autem dicimus omnia fạcta esse a nibilo a deo qui non solum est sed etiam summus e est. Possumus tamen dicere similiter ${ }^{f}$ quod $a^{g}$ nihilo nihil fit, quippe cum omnia fiant ex quattuor elementis, terra aqua aere et $\mathbf{b}$ igne. Si quis autem interroget terra unde facta est uel cetera ${ }^{i}$ elementa, dicendum est, ex nihilo. Principium uero non a se sed a deo principe $j$ sumpserunt. Et ideo forte potest dici elementa de nihilo facta, quod a deo qui summum habet esse creata sunt ${ }^{1}$.

[This gloss is not in $L$ which is mutilated at this point. It is contained in $K, C$, and $F$.]

ib. (p. I 23, 1. 55). Ordo . . procedens. Ambitus dispositionis dei, extra quem et praeter quem nibil usquam aut est aut erit.

V pr. 2 (p. 124, 1. 5). Neque enim fuerit ulla rationalis natura, quia eidem libertas adsit arbitrii. Sciendum itaque quod soli angeli et homines rationabiles a deo creati sunt quibus etiam liberum arbitrium est concessum siue ad bonum siue ad malum, et maligni quidem angeli statim ut praeuaricati sunt liberum perdiderunt arbitrium ita ut bonum iam nec $\mathrm{m}$ uelint nec possint. In malo autem quod eligerunt suum arbitrium tantummodo remansit. Sancti uero $\mathbf{n}$ angeli territi ruina malignorum spirituum ita solidati sunt et inmeliorati in arbitrio solius bonitatis ut iam nec uelint nec etiam possint malum, et bonum quod

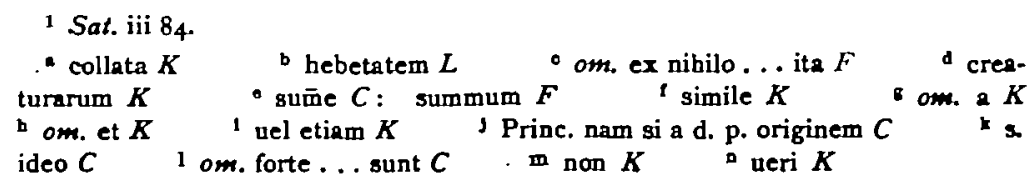


uelint statim possint omnino perficere. Similiter ergo et homo antequam peccaret liberum habuit arbitrium scil. utrum in natura boni permaneret an ad malum rueret. Neque enim repugnabat caro legi mentis illius ad peccatum. ${ }^{1}$ At postquam nullo cogente peccauit, arbitrium bene agendi (penitus perdidit) et ad malum tantum ad quod sponte lapsus est liberum habuit arbitrium. Non solum igitur homo bonum per se non potest agere, sed nec cogitare sine solius dei clementia. 〈(Habet $)\rangle^{a}$ itaque homo liberum arbitrium, non tamen sanum sed corruptum et semper ad malum ruens.

V pr. 2 (p. 1 24, 1. 14). Perspicax iudicium. Perspicax .i. euidens, quia non falluntur sicuti homines uerisimilibus bonis nec aliquid possunt dubitare in rebus eligendis uelut homines. Hinc legitur: sunt uiae quae putantur rectae, quarum finis usque ad profundum inferni demergitur. ${ }^{2}$

ib. (p. 124, 1. 15). Humanas uero animas liberiores quidem esse necesse est, aum se in mentis diuinae speculatione conserwant. Secundum philosophos hoc dicit qui dicunt (animas) esse in contemplatione et suam uim plenissime exercere antequam ad corpora ueniant; cum autem descendant in corpus, obliuisci suae naturae et grauidine ${ }^{b}$ corporis eas opprimi. Unde a quibusdam reprehenditur ${ }^{\circ}$, non tamen reprehendendus $^{d}$ est, cum beatus Augustinus similiter dixerit ${ }^{\theta}$, sed in libro Retractationum se deinde redarguit, dicens tempore $f$ certo animas creari et certo tempore eas in corpore destinari. ${ }^{3}$

V pr. 3 (p. 125, 1. r). Tum ego: En, inquam, difficiliore rursus ambiguitate confundor. Caute et attentissime $B$ legenda sunt ista usque dum ex persona $\mathrm{Philosophiae} \mathrm{respondeatur} \mathrm{quoniam} \mathrm{minus} \mathrm{perfectorum}$ proponit opiniones nec tamen proposita concludit; (plura) etiam neganda interponit. Dicit ergo, deus cuncta praeuidet quae futura sunt cum nihil $\mathbf{h}$ potest falli, et quaecumque praeuidet necesse est ut fiant. Igitur si necesse habent fieri secundum dei prouidentiam, ubi erit arbitrii libertas? $\mathrm{Si}^{\mathrm{i}}$ necessitas est imposita $\mathrm{j}$ ex parte prouidentiae, liberum non erit arbitrium. Sed sciendum quia deus non imponit necessitatem gerendis rebus neque (cogit) ea impleri quae praeuidet, sed sicut gerendae sunt ipsae res per liberum arbitrium, ita deus praeuidet illas futuras. Verbi gratia : si celebretur currule certamen et aliquis in editiori stans loco spectet, hic nulli currentium uires administrat aut quemlibet percurrere facit; aspicit tamen qui uincat et qui melius currat. Sic ergo quodammodo deus non compellit ea quae sunt facienda ad suam uenire prouidentiam, sed praeuidet omnino quo modo uel quando quid

\footnotetext{
1 Rom. vii 23. "Prou. ix 18 , xiv I2, xvi 25. 2 Ratratt. i 8.

- Habet $C F$ b graui $L$ ' reprehenduntur $L$ d reprehendendum $L$

- simile dicit $K \quad t$ redarguit alii autem tempore $K$ intentissime $L$

bui nihil $K \quad{ }^{1}$ Nem si $L \quad$ opposite $L$ : inpos. $K$.
} 
futurum sit, quippe cui nihil • futurum, nihil praeteritum est, sed omnia ei potius praesentia sunt, sicut supra quoque ait, Quae sint, quae fuerint ${ }^{b}$, ueniantque, uno mentis cernit in ictu. Sicut de Ioseph intellegi potest, quem prophetia somnium dixerat futurum dominum fratrum suorum qui cum ei inuiderent uendiderunt eum, ne posset fieri eorum dominus; et ubi fugere conati sunt diuina prouidentia ibi comprehensi sunt. Nam si non uendidissent eum Aegyptiis non posset in Aegypto fieri eorum dominus.

ib. (p. 129, l. Ior). Qui solus modus est etc. Ut enim dicit beatus Augustinus, Quando homines deum orant cum eo loquuntur. ${ }^{1}$ Illique inaccessae luci i. inaccessibili. Beato Paulo concordat dicenti: Qui habitat lucem inaccessibilem,' quod ideo dicitur quia nullus intellectus penetrare eam sufficit. Sicuti hinc (idem) apostolus alibi : Pax dei quae exsuperat omnem sensum, hoc est humanum et angelicum.

V m. 3 (p. 130, 1. 20). An cum mentem cerneret altam (.i. diuinam) in eius scil. positam contemplationem. Philosophi enim dicunt animas antequam corporibus accedant positas esse in contemplatione diuina, et tunc cognoscere omnia quaecumque a deo per species creata sunt; at postquam in corpora descenderint uniuersitatem quidem retinere .i. genus; speccies uero perdere quas necesse est homini in ${ }^{c}$ corpore requirere.

V pr. 4 (p. 134, 1. 80). Aliter intellegentia coniuetur. Quae ipsam rationem transcendit et ad sola spiritalia et diuina se erigit per contemplationem. Postquam enim mens uniuersa perlustrat quae a deo creata sunt, incipit quaerere auctorem ipsum qui ea condidit. Nam per ea quae mirabiliter et diuerso modo creata considerat, intellegit multo mirabiliorem esse auctorem qui tanta et tam miranda sine labore perfecit. Unde apostolus ait: Inuisibilia enim dei per ea quae facta sunt intellecta conspiciuntur ; sempiterna quoque eius uirtus et diuinitas." Hoc ergo per solum fit intellectum. Sciendum uero quia cum simul sint semper ratio et intellegentia, intellegentia tamen in hoc rationem transcendit quia cum homo omnis rationis sit capax, non homo omnis tamen intellegentiae habet sublimitatem ut ${ }^{\mathrm{d}}$ puritatem diuinae maiestatis ualeat contemplari.

V m. 4 (p. I 35, l. I). Quondam porticus attulit $\mid$ obscuros nimium seres. Hoc est, Stoa habuit. Stoa dicitur porticus ; inde stoici philosophi qui dixerunt imagines corporum ita mentibus ${ }^{\theta}$ imprimi sicut in pagina figuntur litterae uel notae. Academia fuit uilla iuxta Athenas ciuitatem sita non plus ab ea distans uno miliario. Interpretatur autem academia populi tristitia eo $\mathrm{f}$ quod ibi Neptunus superatus sit bellico

\footnotetext{
1 Erarr. in Ps. lxxxv 7. 'I Tim. vi 16. 'S Phil. iv 7. $\quad$ Rom. i 20.

- quod cum nihil $L \quad$ b quae sunt quae fuerunt $L$ e necesse habent in $K \quad$ et $K \quad$ menti $L \quad$ et $K$
} 
conflictu, sicut Gregorius Nazianzenus ait pro quodam homicidio quod inter eos contigit. Victus ergo Neptunus turpiter ab eadem uilla nauigio aufugit. In ipsa uilla Plato philosophus commorans omni tempore uitae suae philosophiam docebat. Post cuius obitum scola ipsius in tres sectas diuisa est, et qui in eadem uilla remanserunt ab ea academici sunt dicti. Qui uero exeuntes a uilla ciuitatem Athenas sunt ingressi, Stoici sunt nuncupati, et hi in porticibus philosophabantur ${ }^{\text {. }}$. Stoa enim ${ }^{b}$ graece porticus dicitur latine. Tertia secta est quae ad loca diuersa migrando nullam certam sedem habebat et appellatur peripatetica. Quia igitur huius sectae philosophi diuersa loca peragrabant, peripatetici uocati sunt .i. deambulantes, seu ut Hieronymo placet circumcalcantes eo quod partes orbis circumirent gratia discendarum disciplinarum: quicquid enim diffiniebant uelut stabile quiddam et inconcussum ${ }^{\circ}$ et undique circumcalcatum .i. fixum manebat. Quod enim ${ }^{d}$ nos dicimus calco Graeci dicunt pato. Inde componitur peripato .i. circumcalco; inde peripatetici sunt dicti qui alias sectas altiori ingenio superexcellunt.

V pr. 5 (p. 1 36, 1. 5). Intrinsecus formas. Spiritales animae formas dicit quae excitantur passione sensuum exteriorum; dicit enim Plato omnium rerum formas in anima incomprehensibiliter et inuisibiliter contineri antequam per corporales et exteriores excitentur formas.

V pr. 6 (p. 143, 1. I17). Quaedam de libero proficiscuntur arbitrio .i. mala. Mala non solummodo ${ }^{\theta}$ (libero fiunt arbitrio, quia postquam primus homo sponte peccauit ad bonum quidem liberum amisit arbitrium, ad malum) tantum retinuit. Nisi ergo diuina inspiratio praeueniat, nullus aliquid ualet agere boni. Hinc Apostolus : Plus, inquit, omnibus laboraui, non autem ego sed gratia dei mecum.' Mirum uidetur quod dicit se laborasse et non laborasse $\mathrm{f}$, quod facile potest uideri, si simul et gratiam dei quae illum praeuenit et liberum arbitrium consideremus.

1 I Cor. xv ro.

* philosophantur $K L \quad$ b autem $L$ o concussum $K L$

- solummodo admodum $K$ mala non solum libero sunt arbitrio $L$ The gloss is printed as it stands in $C$ except for ${ }^{\prime}$

H. F. Stewart. 